\title{
Overexpression of serine/threonine-protein kinase-1 in pancreatic cancer tissue: Serine/threonine-protein kinase-1 knockdown increases the chemosensitivity of pancreatic cancer cells
}

\author{
QINGGUANG WANG ${ }^{1 *}$, DONG XU ${ }^{2 *}, \mathrm{CHAO} \mathrm{HAN}^{3}, \mathrm{MIN} \mathrm{TU}^{4}$, QING DU ${ }^{4,5}$, \\ JINGJING ZHANG ${ }^{4,5}$, YI ZHU ${ }^{4,5}$ and LIJIAN XU ${ }^{3}$ \\ ${ }^{1}$ Department of General Surgery, Zoucheng People's Hospital, Jining, Shandong 273500; ${ }^{2}$ Department of General Surgery, \\ Gaochun People's Hospital, Nanjing, Jiangsu 211300; ${ }^{3}$ Department of General Surgery, \\ The Second Affiliated Hospital of Nanjing Medical University, Nanjing, Jiangsu 210011; ${ }^{4}$ Department of General Surgery, \\ The First Affiliated Hospital of Nanjing Medical University, Nanjing, Jiangsu 210029; \\ ${ }^{5}$ Jiangsu Province Academy of Clinical Medicine, Institute of Tumor Biology, Nanjing, Jiangsu 210029, P.R. China
}

Received May 22, 2014; Accepted January 29, 2015

DOI: $10.3892 / \mathrm{mmr} .2015 .3434$

\begin{abstract}
Serine/threonine-protein kinase-1 (SMG-1) belongs to the phosphatidylinositol 3-kinase-related kinase family. Altered expression of SMG-1 contributes to human carcinogenesis and cancer progression. The present study detected the expression levels of SMG-1 in normal and cancerous pancreatic tissues and then assessed the effects of SMG-1-knockdown in pancreatic cancer cell lines in vitro. A pancreatic cancer tissue array and pancreatic cancer cell lines were used to detect the expression levels of SMG-1 and a lentivirus expressing either SMG-1 or negative control short hairpin (sh)RNA were used to knock down the expression of SMG-1 in the pancreatic cancer cell lines. Western blot, cell proliferation, Cell Counting kit-8, Transwell tumor cell migration and invasion assays, and flow cytometric analysis of cell apoptosis with or without gemcitabine or cisplatin treatment were performed to assess the tumor cells. The protein expression of SMG-1 was higher in the pancreatic cancer tissues and cells compared with the normal tissues. sh-SMG-1 lentivirus infection significantly suppressed the expression of SMG-1 in the pancreatic cancer cell lines, resulting in the inhibition of tumor cell proliferation and increased chemosensitivity to treatment with gemcitabine and cisplatin. However, SMG-1 knockdown had no effect on pancreatic cancer cell migration or invasion capacities. The protein expression of SMG-1 was increased in the pancreatic
\end{abstract}

Correspondence to: Dr Lijian Xu, Department of General Surgery, The Second Affiliated Hospital of Nanjing Medical University, 121 Jianjiayuan Road, Nanjing, Jiangsu 210011, P.R. China

E-mail: xulijian185@126.com

*Contributed equally

Key words: pancreatic cancer, serine/threonine-protein kinase 1, short hairpin RNA, cell proliferation, apoptosis cancer tissues and was associated with an advanced tumor stage. Knock down of the expression of SMG-1 inhibited tumor cell proliferation and induced the chemosensitivity of pancreatic cancer cells in vitro.

\section{Introduction}

Pancreatic cancer is a significant health problem worldwide (1), with an estimated 279,000 cases diagnosed globally in 2008 (2). The majority of patients are diagnosed at an advanced stage, and the 5-year survival rate of patients following surgery is poor, with 213,000 pancreatic cancer-associated mortalities worldwide despite advancements in surgery, radiation therapy and chemotherapy (3). Therefore, it is important to identify the molecular mechanisms underlying the development and progression of pancreatic cancer in order to develop novel strategies for its early detection and treatment.

The present study investigated serine/threonine-protein kinase-1 (SMG-1), which belongs to the phosphatidylinositol 3-kinase-related kinase (PIKK) family $(4,5)$. Functionally, SMG-1 has been linked to the nonsense-mediated decay (NMD) of mRNA, as a member of the mRNA surveillance complex (4). SMG-1 also has NMD-independent functions (4), including cellular stress responses. Previous studies $(6,7)$ have demonstrated that hSMG-1 is an important regulator of the cell cycle checkpoints by regulating the synthesis and proteolysis of p21. SMG-1 is also considered to be a potential cancer susceptibility gene. The catalogue of somatic mutations in the COSMIC cancer database reveals that mutations in $S M G-1$ are associated with breast, kidney and stomach cancer (www.sanger.ac.uk/genetics/CGP/cosmic) (8). Previous investigations have demonstrated that SMG-1 is widely expressed in multiple tissues and cell lines $(5,9)$. It has been observed that SMG-1 regulates the G1/S checkpoint of the cell cycle via a p53-dependent and a p53-independent pathway, and depletion of the SMG-1 protein increases the cell growth of colorectal cancer cells, indicating that SMG-1 is a tumor suppressor gene (10). Another study demonstrated that the 
SMG-1-induced activation of the p53 pathway is associated with the chemoprotective effects of tempol (11). However, additional studies have revealed that the $S M G-1$ promoter hypermethylation-induced downregulation of the expression of SMG-1 is associated with improved survival rates in patients with human papaloma virus (HPV)-positive head and neck squamous cell carcinoma (12). SMG-1 antagonizes tumor necrosis factor- $\alpha$-induced apoptosis in osteosarcoma cells (13). A kinome-wide screen identified SMG-1 as an essential kinase for the survival of multiple myeloma and SMG-1 knockdown with small interfering (si)RNA reduced the survival of myeloma cell lines (14). Furthermore, SMG-1 mRNA has been observed to be upregulated in acute myeloid leukemia (15). These studies indicated that SMG-1 may have different roles in cancer progression.

The present study detected the protein expression of SMG-1 in pancreatic cancer tissue specimens and subsequently assessed the effects of SMG-1 knockdown on the sensitivity of pancreatic cancer cells to chemotherapy in vitro.

\section{Materials and methods}

Immunohistochemical analysis of the expression of SMG-1 in pancreatic cancer tissue. The present study was approved by the ethics committee of the Second Affiliated Hospital of Nanjing Medical University (Nanjing, China). A pancreatic cancer tissue microarray (TMA) was obtained from Alenabio (cat. no. PA2082; Xian, China). The TMA contained 94 cases of pathologically diagnosed pancreatic adenocarcinoma with certain additional clinicopathological data from the patients. A pathologist inspected all the specimens and confirmed the diagnosis of pancreatic adenocarcinoma, mucinous adenocarcinoma, adenosquamous carcinoma, squamous cell carcinoma, acinic cell carcinoma and neuroendocrine carcinoma, five cases of normal pancreatic tissue and five cases of distant normal pancreatic tissue. The age of the tissue donors ranged between 39 and 78 years, with a mean age of $57.5 \pm 9.9$ years, and a female to male ratio of 1.6. The tumor tissue was obtained from patients at Tongxu People's Hospital (Kaifeng, China).

For immunostaining of the SMG-1 protein, the TMA sections were deparaffinized in xylene (Xilong Chemical Co., Ltd., Shenzhen, China) twice and rehydrated in a series of ethanol (100\% for $5 \mathrm{~min}, 95 \%$ for $5 \mathrm{~min}, 75 \%$ for $5 \mathrm{~min}$ ) followed by ultrapure water. Staining was performed by incubating the sections with mouse monoclonal anti-SMG-1 antibody (1:300; cat. no. SAB1404950-100UG; Sigma-Aldrich, St. Louis, MO, USA), at $4^{\circ} \mathrm{C}$ overnight. The intensity of the SMG-1 staining was scored by a pathologist, in a blinded manner, as negative (no signal), weak (weak intensity in $<50 \%$ of tumor cells), moderate (strong intensity in $<40 \%$ of tumor cells) or strong (strong intensity in the majority of tumor cells).

Cell lines and culture. The COLO-357, BxPc-3, Capan-1, Capan-2, SW1990 and PANC-1 human pancreatic cancer cell lines were obtained from the Shanghai Cell Bank (Shanghai, China) and cultured in Dulbecco's modified Eagle's medium (DMEM), supplemented with $10 \%$ fetal bovine serum (FBS), $100 \mu \mathrm{g} / \mathrm{ml}$ penicillin and $100 \mu \mathrm{g} / \mathrm{ml}$ streptomycin in a humidified atmosphere of $5 \% \mathrm{CO}_{2}$ at $37^{\circ} \mathrm{C}$. All reagents were obtained from Wisent (St. Bruno, PQ, Canada).

SMG-1 short hairpin (sh)RNA recombinant lentiviral vectors and lentivirus. A lentiviral vector-mediated shRNA against SMG-1 was designed and constructed by GenePharma (Shanghai, China). Four pairs of shRNAs, each targeting different regions of the SMG-1 transcript (GenBank accession no. NM_015092), and one negative control shRNA were constructed. The target mRNA sequences of SMG-1 were as follows: sh-SMG-1 \#1 (no. 5252), 5'-GCAGAAAG GTGGTTGACAATG-3'; sh-SMG-1 \#2 (no. 6931), 5'-GCT CGACACTATTCTGTAACA-3'; sh-SMG-1 \#3 (no. 7512), 5'-GGGTGTAACTGGAGTAGAAGG-3' and sh-SMG-1 \#4 (no. 8877): 5'-GGAAGCGTCTGAGACAGTTCA-3'. The scrambled sequence, 5'-ACTACCGTTGTTATAGGTG-3', was used as a negative control. These lentiviral vectors were then used by GenePharma to produce the lentivirus.

Lentivirus infection of pancreatic cancer cell lines. To knock down the expression of SMG-1, the SW1990 and PANC-1 pancreatic cancer cell lines were infected with a lentivirus. Briefly, the cells were seeded into six-well plates (Corning Costar, Inc., Corning, NY, USA) at a density of $40 \%$ and grown for $24 \mathrm{~h}$ at $37^{\circ} \mathrm{C}$. The cells were then infected with a lentivirus containing the shRNA targeting SMG-1[SW1990 at an multiplicity of infection (MOI) of 10 and PANC-1 at an MOI of 15, based on pre-experimental data], according to the manufacturer's instructions. The lentiviral infection efficiency was confirmed by the immunofluorescence density of the enhanced green fluorescent protein reporter gene, and the RNA interference (i) efficiency was determined by analyzing the mRNA and protein expression of SMG-1. Based on these investigations, SMG-1 shRNA lentivirus construct \#1 was selected for the subsequent experiments (data not shown).

$R N A$ isolation and reverse transcription-quantitative polymerase chain reaction ( $R T-q P C R)$. The cells were washed with phosphate-buffered saline (PBS; Beyotime Institute of Biotechnology, Haimen, China) and the total RNA was isolated from the cells using RNAiso Plus (Takara Bio, Inc., Dalian, China), according to the manufacturer's instructions. Primer-Script RT Master mix (Takara Bio, Inc.) was used to synthesize cDNA from the RNA samples.qPCR was performed using SYBR Green (Roche Diagnostics, Indianapolis, IN, USA) on a 7500 Real-Time-PCR System (Applied Biosystems, Foster City, CA, USA). The qPCR amplification was performed on $10 \mathrm{ng}$ cDNA (total reaction volume, $20 \mu \mathrm{l}$ ) as follows: An initial cycle of $95^{\circ} \mathrm{C}$ for $10 \mathrm{~min}, 40$ cycles of $94^{\circ} \mathrm{C}$ for $30 \mathrm{sec}$, $60^{\circ} \mathrm{C}$ for $30 \mathrm{sec}$ and $72^{\circ} \mathrm{C}$ for $30 \mathrm{sec}$, and then a final extension at $72^{\circ} \mathrm{C}$ for $5 \mathrm{~min}$. The mRNA expression of $\beta$-actin was used as an internal control for determining the relative mRNA expression of SMG-1. The cycle threshold $(\mathrm{Ct})$ comparative $\Delta \Delta \mathrm{Ct}$ method was used to calculate the relative mRNA expression of SMG-1, and the fold-changes were analyzed by $2^{-\Delta \Delta C t}(16)$. The primers used for RT-qPCR were as follows: SMG-1, forward 5'-TTAATCGCCAAGAAACACCC-3' and reverse 5'-AGGAATCTTGGGCCTTTTGT-3' and $\beta$-actin, forward 5'-CTCCATCCTGGCCTCGCT-3' and reverse 5'-GCTGTCACCTTCACCGTTCC-3'. All the experiments 
were performed in triplicate and repeated three times with independent RNA samples.

Protein extraction and western blotting. The total cellular protein was extracted from the SW1990 and PANC-1 cells using radioimmunoprecipitation buffer (Beyotime Institute of Biotechnology), supplemented with $1 \%$ phenylmethylsulfonylfluoride (Beyotime Institute of Biotechnology). The protein concentration was estimated with a (bicinchoninic acid) BCA kit (Nanjing KeyGen Biotech. Co., Ltd., Nanjing, China). Following quantification, the protein samples were separated by $6 \%$ sodium dodecyl sulfate polyacrylamide gel electrophoresis (Beyotime Institute of Biotechnology) and transferred onto polyvinylidenedifluoride membranes (Beyotime Institute of Biotechnology). The membranes were blocked with 5\% non-fat dry milk (Yili Industrial Group Co., Ltd., Inner Mongolia, China) in Tris-buffered saline (TBS) and incubated with the following primary antibodies: mouse monoclonal anti-SMG-1 (1:300; cat. no. SAB1404950-100UG; Sigma-Aldrich) or mouse monoclonal anti-GAPDH (1:500; cat. no. AG019; Beyotime Institute of Biotechnology, Jiangsu, China) at $4^{\circ} \mathrm{C}$ overnight. The following day, the membranes were washed with TBS-Tween-20 (TBS-T; Beyotime Institute of Biotechnology) and further incubated with a secondary horseradish peroxidase-coupled goat anti-mouse antibody (Santa Cruz Biotechnology, Inc., Santa Cruz, CA, USA) for $2 \mathrm{~h}$ at room temperature. The membranes were washed three times with TBS-T and the color was developed using an electrochemiluminescence kit (Pierce Biotechnology, Inc., Rockford, IL, USA). The membranes were then exposed to X-ray film (GelDoc XR system; Bio-Rad Laboratories, Inc., Hercules, CA, USA) to visualize the signals. The target protein expression of GAPDH was used as an internal control for determining the relative expression levels of SMG-1.

Cell proliferation assay. The cells were infected with either the negative control lentivirus or the SMG-1 shRNA lentivirus for 3 days at $37^{\circ} \mathrm{C}$ and then seeded into 96 -well culture plates (Costar, Cambridge, UK) at a density of $2 \times 10^{3}$ cells/well. The cells were incubated at $37^{\circ} \mathrm{C}$ for up to 5 days. Cell proliferation was detected daily using a Cell Counting kit-8 (CCK-8; Nanjing KeyGen Biotech., Co., Ltd.), according to the manufacturer's instructions, for 5 days. Briefly, $10 \mu \mathrm{l} \mathrm{CCK-8} \mathrm{solution}$ was added to each well and the optical density was detected using a microplate reader (Sunrise ${ }^{\mathrm{TM}}$; Tecan, Grödig, Austria) at $450 \mathrm{~nm}$, with a reference wavelength of $650 \mathrm{~nm}$. Each assay was performed in triplicate and repeated independently three times.

Tumor cell migration and invasion assay. The tumor cell migration and invasion capacities were measured using a Transwell chamber assay with or without Matrigel coating. The Transwell chambers were $6.5 \mathrm{~mm}$ in diameter and had a $8 \mu \mathrm{m}$ pore size (Corning Costar, Inc.). The SW1990 and PANC-1 cells were seeded into the upper chamber $\left(5.0 \times 10^{4}\right.$ cells per Transwell) pre-coated with or without $1 \mathrm{mg} / \mathrm{ml}$ Matrigel (BD Biosciences, San Jose, CA, USA), and the lower wells were filled with $500 \mu 1$ 10\% FBS-DMEM. Following incubation for $24 \mathrm{~h}$ at $37^{\circ} \mathrm{C}$, the non-invading cells were removed using cotton swabs and the cells that had
A

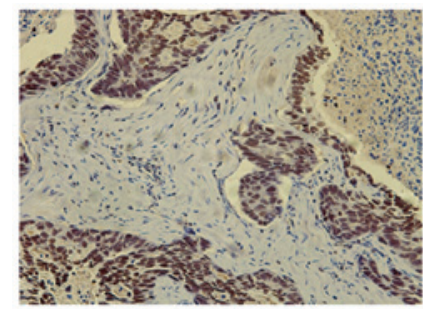

B

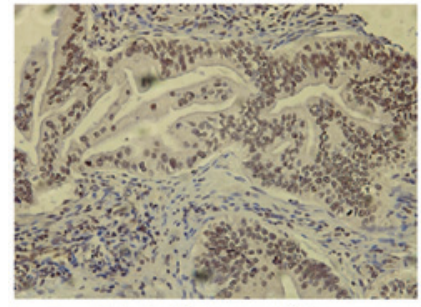

C

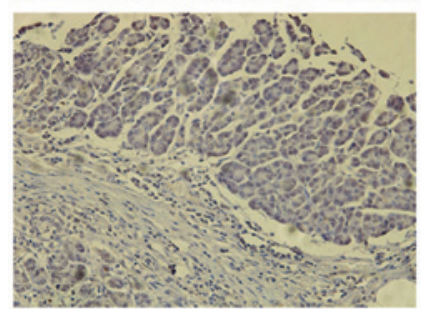

D

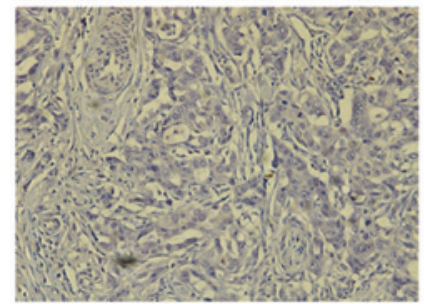

Figure 1. Differential protein expression levels of SMG-1 in pancreatic cancer from a tissue microarray (hematoxylin and eosin stain) with immunohistochemical staining. Representative images reveal (A) strong, (B) moderate, (C) weak and (D) negative protein staining of SMG-1 in the pancreatic cancer tissue. Magnification, x200. SMG-1, serine/threonine-protein kinase-1.

invaded into the underside of the membrane were stained with $0.1 \%$ crystal violet (Beyotime Institute of Biotechnology) for $15 \mathrm{~min}$ at $37^{\circ} \mathrm{C}$. The membranes were washed with PBS and the invading cells were counted under an inverted microscope (Eclipse Ti-E; Nikon Corporation, Tokyo, Japan). All the experiments were performed in triplicate and repeated once.

Flow cytometric analysis of cell cycle distribution and apoptosis. The cell cycle progression and apoptosis were assessed by flow cytometry using a BD FACSCalibur (BD Biosciences). The SW1990 and PANC-1 cells were grown and infected with sh-SMG-1 or with a control lentivirus at $37^{\circ} \mathrm{C}$ for $12 \mathrm{~h}$, and then treated with $10 \mathrm{mg} / \mathrm{ml}$ gemcitabine or cisplatin (Jiangsu Hansoh Pharmaceutical Co., Ltd., Jiangsu, China). Cell cycle analysis was conducted using PI/RNase Staining Buffer (BD Pharmingen, San Diego, CA, USA). For cell cycle analysis, the cells were collected, washed twice with PBS and fixed with $70 \%$ ethanol at $-20^{\circ} \mathrm{C}$ overnight. The cells were then washed twice with PBS and resuspended in $500 \mu \mathrm{l}$ PBS containing $0.2 \%$ Triton-X-100, $10 \mathrm{mM}$ EDTA, $100 \mu \mathrm{g} / \mathrm{ml}$ RNase A and $50 \mu \mathrm{g} / \mathrm{ml}$ propidium iodide. The samples were incubated at room temperature for $30 \mathrm{~min}$. 
Table I. Association of the expression of serine/threonine-protein kinase-1 with clinicopathological data from the patients.

Expression level of SMG-1

\begin{tabular}{|c|c|c|c|}
\hline \multirow[b]{2}{*}{ Group } & \multirow{2}{*}{ Total (n) } & \multirow{2}{*}{ Low (n) } & \multirow{2}{*}{ High (n) } \\
\hline & & & \\
\hline \multicolumn{4}{|l|}{ Gender } \\
\hline Female & 36 & 16 & 20 \\
\hline Male & 58 & 30 & 28 \\
\hline \multicolumn{4}{|l|}{ Age (years) } \\
\hline 45 & 11 & 5 & 6 \\
\hline $45-60$ & 53 & 25 & 28 \\
\hline 60 & 30 & 16 & 14 \\
\hline \multicolumn{4}{|l|}{ Pathology type } \\
\hline Ductal adenocarcinoma & 83 & 43 & 40 \\
\hline Others & 11 & 4 & 7 \\
\hline \multicolumn{4}{|l|}{ TNM grading $^{\mathrm{a}}$} \\
\hline I & 31 & 21 & 10 \\
\hline II & 45 & 18 & 27 \\
\hline III & 7 & 3 & 4 \\
\hline $\mathrm{N} / \mathrm{A}$ & 11 & 4 & 7 \\
\hline
\end{tabular}

${ }^{\mathrm{a}} \mathrm{P}=0.026$, I (T1N0M0, T1NxM0 and TxN0M0), vs. II (T2N0M0, T2NxM0 and T3N0M0), vs. III (T-N1M0) and IV (T-N-M1). SMG-1, serine/threonine-protein kinase-1; TNM, tumor-node-metastasis.

Apoptosis analysis was conducted using Annexin V-FITC Apoptosis Detection kit (BD Pharmingen). For the detection of apoptosis, the cells were collected and washed twice with PBS, prior to suspending in $100 \mu 11 \mathrm{X}$ binding buffer and staining with $5 \mu 1$ annexin-V 647 and $5 \mu 1$ 7-aminoactinomycin D at room temperature for $15 \mathrm{~min}$ in the dark. The samples were analyzed using a flow cytometer (BD FACSCalibur; BD Biosciences). All the experiments were performed in triplicate and repeated once.

Statistical analysis. All data are expressed as the mean \pm standard deviation. Student's t-test was used to analyze the differences between groups using SPSS 16.0 software (SPSS, Inc., Chicago, IL, USA). The differences among the strips from the western blot analysis were inferred by comparing the gray level of the strips using ImageJ software, version 1.43 (SeekBio, Huzhou, China). $\mathrm{P}<0.05$ was considered to indicate a statistically significant difference.

\section{Results}

Differential protein expression of SMG-1 in pancreatic cancer tissues. The present study first assessed the protein expression levels of SMG-1 in normal and cancerous pancreatic tissues. Immunohistochemical staining detected SMG-1 in the nuclei and cytoplasm, but was more predominant in the nuclei of the tumor cells (Fig. 1). According to the expression of SMG-1, the pancreatic cancer cases were divided those exhibiting high (moderate/strong) and low (negative/weak) expression levels. In total, $51.1 \%$ (48/94) of the pancreatic cancer tissues expressed a high level of SMG-1 protein, whereas 48.9\% (46/94) expressed a low level of SMG-1 protein (Table I). By contrast, 2/5 distant pancreatic tissues and 1/5 normal pancreatic tissues expressed
A

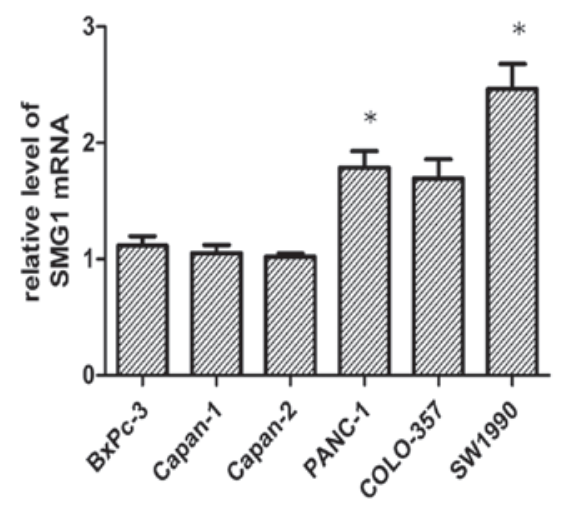

B

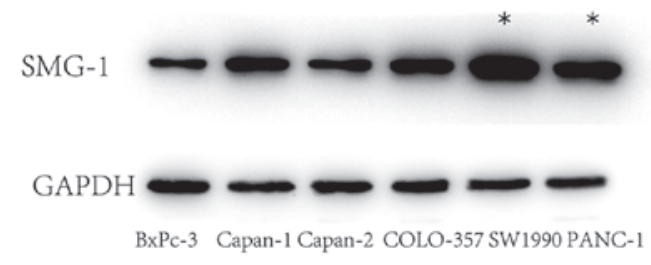

C

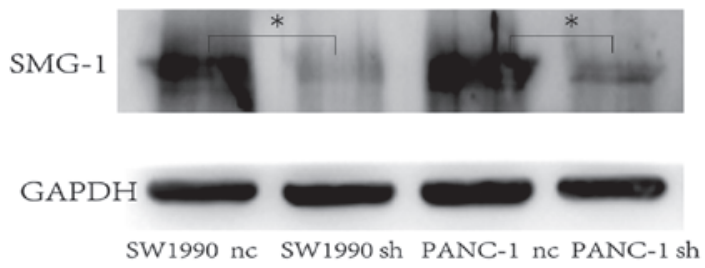

Figure 2. (A) Protein expression levels of SMG-1 in the pancreatic cancer cell lines. Data are expressed as the mean \pm standard deviation. *Denotes 2 cell lines with higher expression of SMG-1. (B) Western blot analysis of the protein expression levels of SMG-1 in the pancreatic cancer cell lines ('denotes 2 cell lines with higher expression of SMG-1) and (C) following knock down of the expression of SMG-1 in the SW1990 and PANC-1 cell lines using SMG-1 shRNA lentivirus \#1. ${ }^{*} \mathrm{P}<0.05$ vs. the $\mathrm{X}$-nc cells. SMG-1, serine/threonine-protein kinase-1; sh, short-hairpin. 
A

B

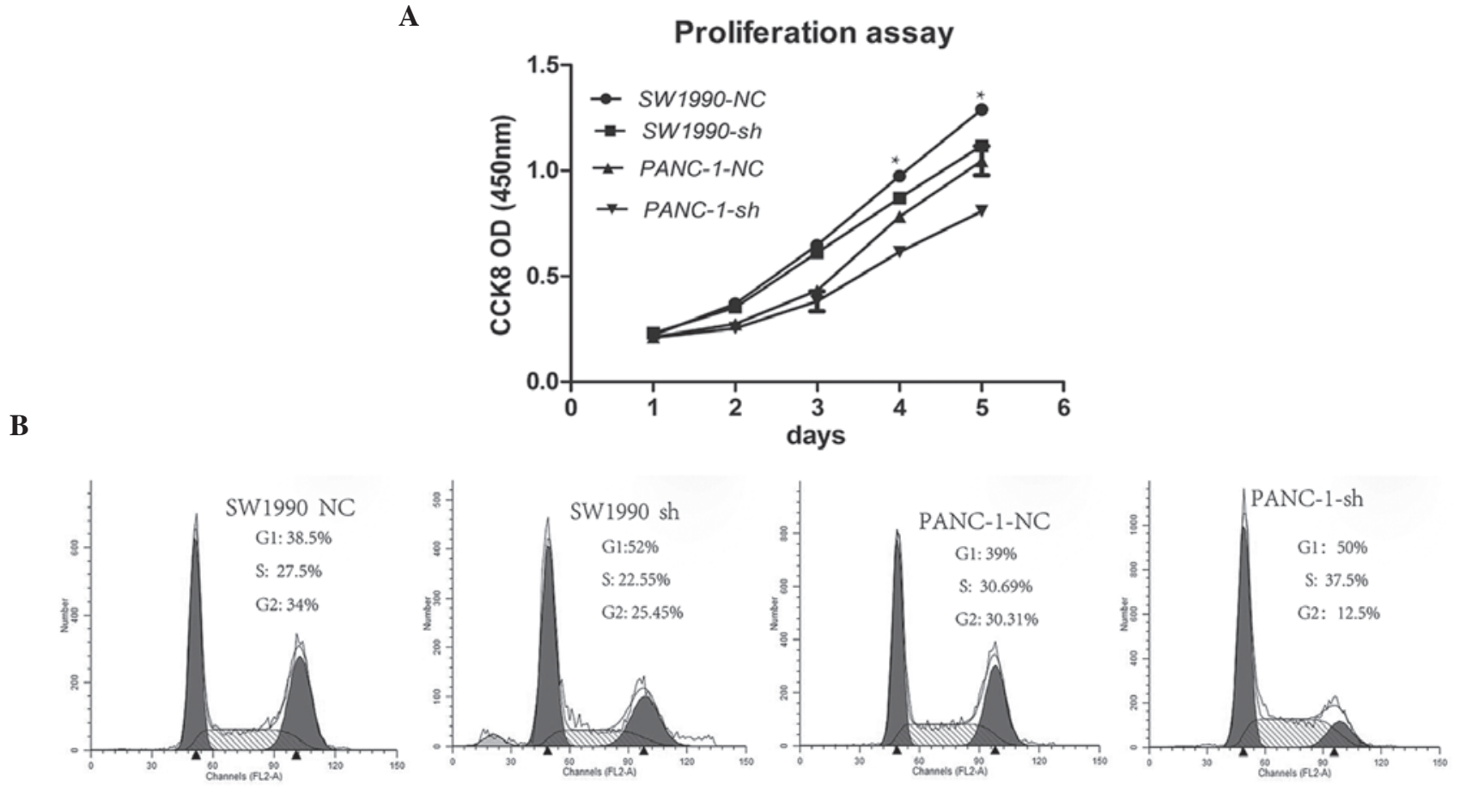

Figure 3. Effect of SMG-1 knockdown on the regulation of pancreatic cancer cell proliferation. (A) Proliferation capacities of the SW1990 and PANC-1 cells were detected using a CCK-8 assay. Data are expressed as the mean \pm standard deviation. The CCK-8 OD of the SW1990 and PANC-1 cells compared with the corresponding X-sh cells showed significant differences on days 4 and 5 ( $\mathrm{P}<0.05)$. (B) Flow cytometric analysis was used to detect the cell cycle progression of the SW1990 and PANC-1 cells. SMG-1, serine/threonine-protein kinase 1; CCK-8, cell counting kit-8; OD, optical density; NC, negative control; sh, short hairpin.

a high level of SMG-1 protein. These results were consistent with those in the Human Protein Atlas (http://www.proteinatlas.org/ENSG00000157106). Since the TMA was obtained from a company with limited clinicopathological data, the expression of SMG-1 was correlated with the expression of SMG-1 among pancreatic cancer tissue, distant pancreatic tissue and normal pancreatic tissue, which revealed that the expression of SMG-1 was associated with an advanced tumor stage, but not with age and gender (Table I).

Expression and knockdown of SMG-1 in pancreatic cancer cell lines. The expression levels of SMG-1 in six different pancreatic cancer cell lines were assessed by western blot analysis. The data revealed that all six pancreatic cancer cell lines expressed high protein levels of SMG-1, and these levels were highest in the PANC-1 and SW1990 cells (Fig. 2A).

Thus, PANC-1 and SW1990 cells were selected for the subsequent knock down of SMG-1 expression using the shRNA lentivirus. The data revealed that SMG-1 shRNA lentivirus \#1 significantly reduced the protein expression levels of SMG-1 in the twi cell lines (Fig. 2B) compared with the negative control shRNA lentivirus.

Knockdown of SMG-1 inhibits the proliferation of pancreatic cancer cells. As shown in Fig. 3A, knockdown of SMG-1 significantly inhibited the proliferation of PANC-1 and SW1990 cells compared with negative control lentivirus-infected tumor cells $(\mathrm{P}<0.05$, vs. control). Flow cytometric analysis revealed that the knockdown of SMG-1 reduced the S/G2 phase of the cell cycle compared with the control cells in the SW1990 cells $(61.67 \pm 2.25$, vs. $44.3 \pm 3.5 \%, \mathrm{P}<0.05)$ and
PANC-1 cells $(61 \pm 4$, vs. $49.7 \pm 3.5 \%$, $\mathrm{P}<0.05$; Fig. $3 \mathrm{~B})$, while the G1 phase of the cell cycle was increased in the SW1990 cells $(38.3 \pm 2.25$, vs. $52.3 \pm 3.5 \%, \mathrm{P}<0.05)$ and PANC-1 cells (39 \pm 4 , vs. $50.3 \pm 3.5 \%, \mathrm{P}<0.05$; Fig. 3B). These observations demonstrated that loss of the expression of SMG-1 inhibited the proliferation of pancreatic cancer cells via the induction of the G1 phase of the cell cycle.

Effect of SMG-1-knockdown on the regulation of pancreatic cancer cell invasion and migration. To determine whether SMG-1 knockdown affected the invasion and migration of the SW1990 and PANC-1 cells, Matrigel invasion and Transwell assays were performed. Infection with the SMG-1 shRNA lentivirus \#1 had no significant effect on the tumor cell invasion and migration capacities (Fig. 4A and B) compared with the cells infected with the control lentivirus.

SMG-1 knockdown increases the chemosensitivity of pancreatic cancer cells to treatment with gemcitabine and cisplatin. Following infection of the pancreatic cancer cells with SMG-1 shRNA and negative control lentiviruses for $72 \mathrm{~h}$, the cells were treated with $10 \mathrm{mg} / \mathrm{ml}$ gemcitabine or $10 \mathrm{mg} / \mathrm{ml}$ cisplatin for an additional $24 \mathrm{~h}$. Flow cytometric analysis revealed that the levels of apoptosis in the SW1990 cells treated with gemcitabine/cisplatin increased, between $16.55 \pm 1.26$ and $21.97 \pm 1.55 \%$ in the gemcitabine-treated cells compared with control cells, and between $9.28 \pm 0.34$ and $16.93 \pm 0.66 \%$ in the cisplatin-treated cells (Fig. 4C and D). Similarly, the PANC-1 cells demonstrated similar responses when treated with gemcitabine $(8.94 \pm 0.59$, vs. $12.35 \pm 0.66 \%$ and cisplatin (10.8 \pm 0.7 , vs. $15.74 \pm 0.89 \%$; Fig. $4 \mathrm{C}$ and E). 
A
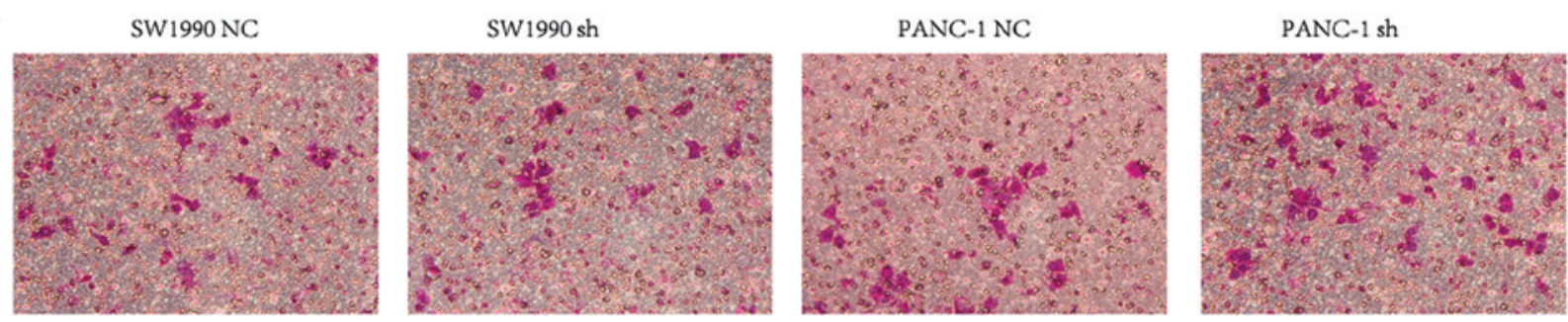

B

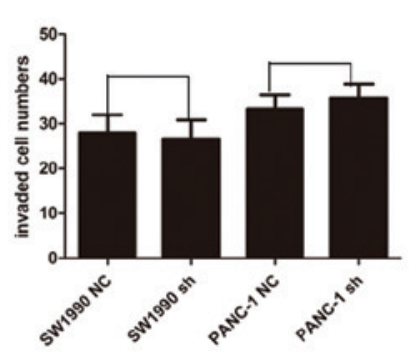

C

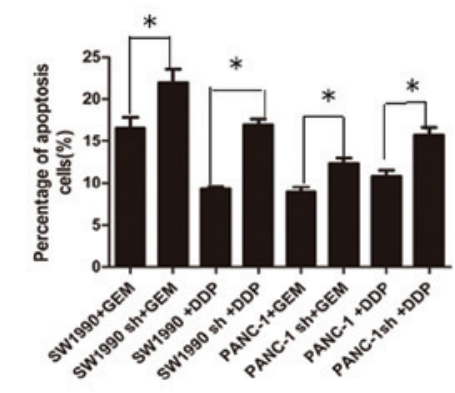

D
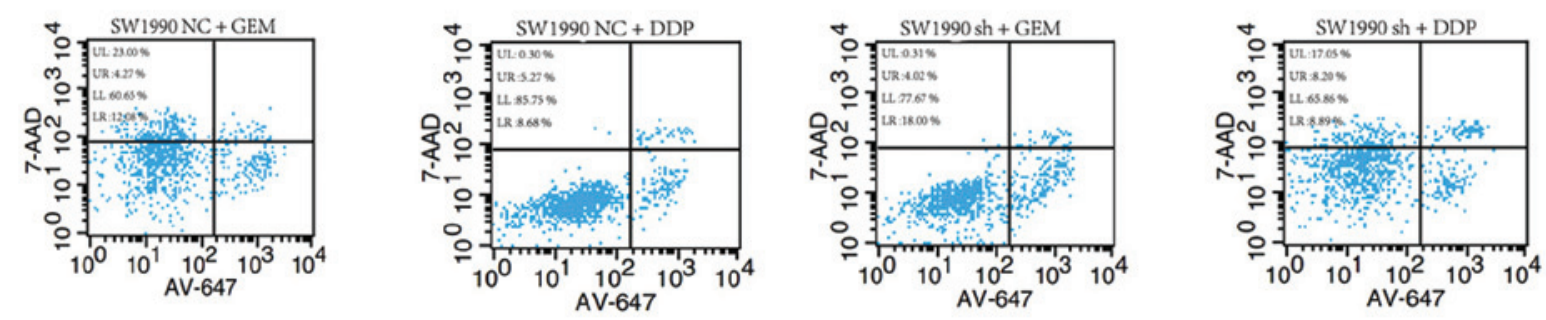

$\mathbf{E}$
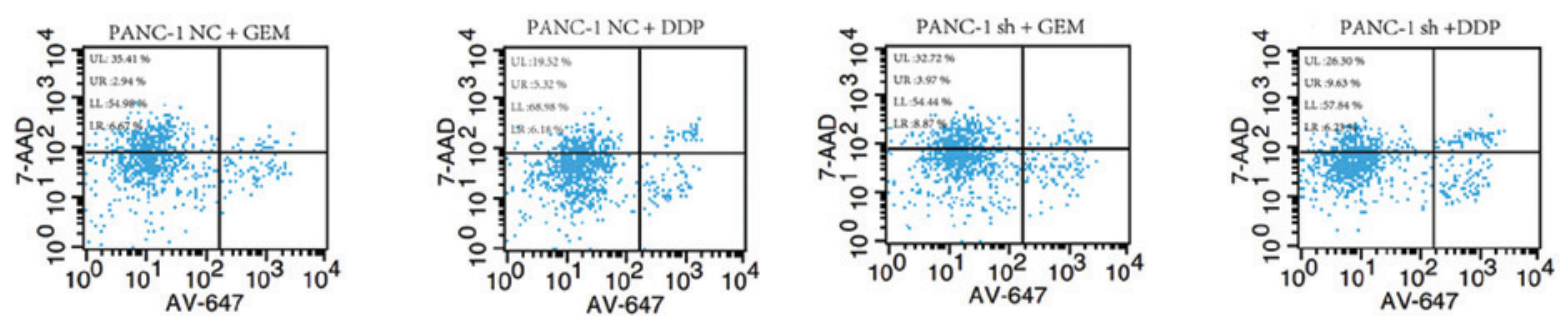

Figure 4. Effect of SMG-1 knockdown on the regulation of pancreatic cancer cell invasion and sensitivity to chemotherapeutic drugs. (A) Representative images of tumor cell invasion and migration (magnification, $\mathrm{x} 200 ; 0.1 \%$ crystal violet stain). (B) Summarized data from (A) based on three independent experiments. (C) Apoptosis of the pancreatic cancer cell lines following infection with the shSMG-1 or control lentivirus $\left({ }^{*} \mathrm{P}<0.05\right.$, vs. control). Data are expressed as the mean \pm standard deviation. (D and E) Changes in tumor cell apoptotic rate. LR+UR is the total apoptotic rate. SMG-1, serine/threonine-protein kinase 1; NC, negative control; sh, short-hairpin; GEM, gemcitabine; DDP, cisplatin; 7-AAD, 7-aminoactinomycin D. UL, upper left; UR, upper right; LL, lower left; LR, lower right.

\section{Discussion}

The present study detected the protein expression levels of SMG-1 in pancreatic cancer and normal tissues, and found that the expression levels of SMG-1 were increased in pancreatic cancer tissue compared with normal tissue. This finding is consistent with data from the Human Protein Atlas. It was also revealed that the protein expression of SMG-1 was associated with an advanced tumor stage. Subsequently, the expression of SMG-1 was knocked down in pancreatic cancer cell lines and phenotypic changes in the tumor cells were observed. The data demonstrated that knock down of SMG-1 inhibited the proliferation of pancreatic cancer cells and increased tumor cell chemosensitivity. However, the tumor cell invasion and migration capacities remained unaltered.

SMG-1 is the newest member of the PIKK family $(4,5)$ due to its homology with CeSMG-1 (5). SMG-1 is involved in NMD of mRNA, and previous studies have demonstrated that abnormal SMG-1 function is involved in human cancer $(5,8,17)$. Other investigations have revealed that SMG-1 is important in human carcinogenesis and cancer progression $(12,18,19)$, and may be a tumor suppressor gene $(5,8,9,20,21)$. These studies demonstrated that SMG-1 is a stress-responsive enzyme and regulates the cell cycle G1/S checkpoint, while silencing of SMG-1 increases tumor cell growth $(10,21)$. By contrast, 
downregulation of the expression of SMG-1 is associated with improved prognosis in head and neck cancer (12).

In the present study, high protein expression levels of SMG-1 were found in pancreatic cancer tissues and cell lines. In addition, the protein expression of SMG-1 was associated with an advanced tumor stage, although only limited clinicpathological data was available from the TMA company. These findings were consistent with data from the Human Protein Atlas.

Previous studies have demonstrated that SMG-1 is involved in multiple biological processes, including cell proliferation, apoptosis and stress responses $(6,13,20,22,23)$. The present study utilized an shRNA technique to knock down the expression of SMG-1 to assess the functions of SMG-1 in pancreatic cancer cell lines (24). The SMG-1 shRNA lentivirus \#1 significantly reduced the expression of SMG-1 in two pancreatic cancer cell lines (Fig. 2B). SMG-1 knockdown inhibited cell proliferation and increased the chemosensitivity of the cells to gemcitabine and cisplatin treatment in vitro. Previous studies have reported that the loss of SMG-1 function significantly increases the rate and extent of apoptotic tumor cell death induced by chemotherapy, irradiation or cytokine treatment $(12,13)$. Another study demonstrated that SMG-1-depleted human cells exhibit an increased level of spontaneous DNA damage (7).

Since the majority pancreatic cancer patients $(\sim 75 \%)$ are diagnosed at an advanced stage, chemotherapy is a tentative treatment option (25). SMG-1 shRNA may assist in treating patients with chemotherapy-resistant tumors. A previous study demonstrated that human papillomavirus (HPV) head and neck cancer cells and tissues express SMG-1 at lower levels compared with HPV-negative cancer tissues, and depletion of SMG-1 in HPV-negative head and neck cancer cells increases the sensitivity to radiation and chemotherapy (12). Another study reported that the sensitivity of lung cancer cells to gemcitabine and cisplatin increased following silencing of the expression of SMG-1 using siRNA (26). The present study demonstrated similar results in pancreatic cancer tissues and cell lines. However, this was only proof-of-principle and further studies are required to fully elucidate the role of SMG-1 protein in the development and progression of pancreatic cancer, by investigating the in vivo effects of SMG-1 knockdown on the chemosensitivity of pancreatic cancer cells.

\section{Acknowledgements}

The authors would like to thank Dr Yi Miao and Dr Min Tu of the Department of General Surgery (Nanjing Medical University, Nanjing, China) for providing assistance and technical support. The authors would also like to thank Medjaden Bioscience, Ltd. (Hong Kong, China) for assisting in the preparation of this manuscript.

\section{References}

1. Yadav D and Lowenfels AB: The epidemiology of pancreatitis and pancreatic cancer. Gastroenterology 144: 1252-1261, 2013.

2. Ghadirian P, Lynch HT and Krewski D: Epidemiology of pancreatic cancer: an overview. Cancer Detect Prev 27: 87-93, 2003.

3. Han H and Von Hoff DD: SnapShot: pancreatic cancer. Cancer Cell 23: 424-424, 2013
4. Yamashita A, Ohnishi T, Kashima I, Taya Y and Ohno S: Human SMG-1, a novel phosphatidylinositol 3-kinase-related protein kinase, associates with components of the mRNA surveillance complex and is involved in the regulation of nonsense-mediated mRNA decay. Genes Dev 15: 2215-2228, 2001.

5. Denning G, Jamieson L, Maquat LE, Thompson EA and Fields AP: Cloning of a novel phosphatidylinositol kinase-related kinase: characterization of the human SMG-1 RNA surveillance protein. J Biol Chem 276: 22709-22714, 2001.

6. Gehen SC, Staversky RJ, Bambara RA, Keng PC and O'Reilly MA: hSMG-1 and ATM sequentially and independently regulate the G1 checkpoint during oxidative stress. Oncogene 27: 4065-4074, 2008.

7. Brumbaugh KM, Otterness DM, Geisen C, et al: The mRNA surveillance protein hSMG-1 functions in genotoxic stress response pathways in mammalian cells. Mol Cell 14: 585-598, 2004.

8. Greenman C, Stephens P, Smith R, et al: Patterns of somatic mutation in human cancer genomes. Nature 446: 153-158, 2007.

9. Uhlen M, Oksvold P, Fagerberg L, et al: Towards a knowledge-based Human Protein Atlas. Nat Biotechnol 28: $1248-1250,2010$

10. Gubanova E, Issaeva N, Gokturk C, Djureinovic $\mathrm{T}$ and Helleday T: SMG-1 suppresses CDK2 and tumor growth by regulating both the p53 and Cdc25A signaling pathways. Cell Cycle 12: 3770-3780, 2013.

11. Erker L, Schubert R, Yakushiji H, et al: Cancer chemoprevention by the antioxidant tempol acts partially via the p53 tumor suppressor. Hum Mol Genet 14: 1699-1708, 2005.

12. Gubanova E, Brown B, Ivanov SV, et al: Downregulation of SMG-1 in HPV-positive head and neck squamous cell carcinoma due to promoter hypermethylation correlates with improved survival. Clin Cancer Res 18: 1257-1267, 2012.

13. Oliveira V, Romanow WJ, Geisen C, et al: A protective role for the human SMG-1 kinase against tumor necrosis factor- $\alpha$-induced apoptosis. J Biol Chem 283: 13174-13184, 2008.

14. Tiedemann RE, Zhu YX, Schmidt J, et al: Kinome-wide RNAi studies in human multiple myeloma identify vulnerable kinase targets, including a lymphoid-restricted kinase, GRK6. Blood 115: 1594-1604, 2010.

15. Neben K, Schnittger S, Brors B, et al: Distinct gene expression patterns associated with FLT3- and NRAS-activating mutations in acute myeloid leukemia with normal karyotype. Oncogene 24: 1580-1588, 2005

16. Livak KJ and Schmittgen TD : Analysis of relative gene expression data using real-time quantitative PCR and the 2(-Delta Delta C(T)) Method. Methods 25: 402-408, 2001.

17. Chen RQ, Yang QK, Chen YL, et al: Kinome siRNA screen identifies SMG-1 as a negative regulator of hypoxia-inducible factor-1 $\alpha$ in hypoxia. J Biol Chem 284: 16752-16758, 2009.

18. Noensie EN and Dietz HC: A strategy for disease gene identification through nonsense-mediated mRNA decay inhibition. Nat Biotechnol 19: 434-439, 2001.

19. Perrin-Vidoz L, Sinilnikova OM, Stoppa-Lyonnet D, Lenoir GM and Mazoyer S: The nonsense-mediated mRNA decay pathway triggers degradation of most BRCA1 mRNAs bearing premature termination codons. Hum Mol Genet 11: 2805-2814, 2002.

20. González-Estévez C, Felix DA, Smith MD, et al: SMG-1 and mTORC1 act antagonistically to regulate response to injury and growth in planarians. PLoS Genet 8: e1002619, 2012.

21. Roberts TL, Ho U, Luff J, et al: Smg1 haploinsufficiency predisposes to tumor formation and inflammation. Proc Natl Acad Sci USA 110: E285-294, 2013.

22. Masse I, Molin L, Mouchiroud L, et al: A novel role for the SMG-1 kinase in lifespan and oxidative stress resistance in Caenorhabditis elegans. PLoS One 3: e3354, 2008.

23. Abraham RT: PI 3-kinase related kinases: 'big' players in stress-induced signaling pathways. DNA Repair (Amst) 3: 883-887, 2004

24. Brummelkamp TR, Bernards R and Agami R: A system for stable expression of short interfering RNAs in mammalian cells. Science 296: 550-553, 2002.

25. Philip PA: Targeted therapies for pancreatic cancer. Gastrointest Cancer Res 2: S16-S19, 2008.

26. Xia QS, Li YZ, Li HY, et al: Effect of inhibiting the expression of hSMG-1 on chemosensitivity of human non-small cell lung cancer H1299 cells. Zhonghua Yi Xue Za Zhi 91: 554-559, 2011 (In Chinese). 